\title{
Resources needed for non-unitary quantum operations
}

\author{
Raam Uzdin \\ Institute for Theoretical Atomic, Molecular and Optical Physics, \\ Harvard-Smithsonian Center for Astrophysics \\ Present address: Racah Institute of Physics, The Hebrew University, Jerusalem, \\ Israel. \\ E-mail: raam@mail.huji.ac.il
}

\begin{abstract}
Non-unitary operations generated by an effective non-Hermitian Hamiltonian can be used to create quantum state manipulations which are impossible in Hermitian quantum mechanics. These operations include state preparation (or cooling) and non-orthogonal state discrimination. In this work we put a lower bound on the resources needed for the construction of some given non-unitary evolution. Passive systems are studied in detail and a general feature of such a system is derived. After interpreting our results using the singular value decomposition, several examples are studied analytically. In particular, we put a lower bound on the resources needed for non-Hermitian state preparation and non-orthogonal state discrimination.
\end{abstract}


Resources needed for non-unitary quantum operations

\section{Introduction}

Unitary quantum evolution generated by Hermitian Hamiltonian conserves the angle between states. For example, two states which are orthogonal at one instant will remain orthogonal throughout the evolution. In effective models of some sub-systems the Hamiltonian may be non-Hermitian. This may be either due to leakage of particles outside the region of interest, (as happens, for example, in Stark ionization), or due to absorption or gain, as often occurs in optics. Another source of non-unitarity can appear when some measurements are performed on entangled systems (e.g [1]). For additional information see 2]. Effective non-Hermitian Hamiltonians lead to nonunitary evolution operators. It was suggested in [3] that non-Hermitian Hamiltonian can be used for perfect discrimination of two non-orthogonal states. In principle this scheme can work even if the states are almost perfectly parallel to each other. In 3 the question of the needed resources for this task was not addressed. In this work, we have fixed a lower bound on the resources needed for a non-unitary task in order to address this question.

We start in Sec. 2 by introducing the "norm action" as a plausible measure for the resources needed to generate some evolution. In Sec. 3 we derive our basic result: a lower bound on the norm action that depends only on the evolution operator at the end of the process. That is, a simple bound can be set on the desired evolution resources without knowing anything on the Hamiltonian. Passive systems are discussed in sec. 4 where our basic result is generalized so that it will be effective for passive systems as well. In Sec. 5 we use the singular value decomposition to explain in what sense our bound quantifies solely the "purely non-unitary" part of the evolution. Next, in section [6] we analytically study several examples in order to illustrate various points concerning our main result. Section 7 contains concluding remarks and the three appendices contain some clarification.

\section{Quantifying evolution resources}

Let $U(t) \in \mathbb{C}^{N \times N}$ be some some non-unitary evolution operator in an $N$-level quantum system. The action of $U(t)$ on a state $|\psi(t=0)\rangle$ is:

$$
|\psi(t)\rangle=U(t)|\psi(0)\rangle \text {. }
$$

In general, the determinant of $U(t)$ is not fixed in time, and it satisfies the Liouville formula [4]:

$$
\operatorname{det}[U(t)]=\operatorname{det}[U(0)] e^{-i \int_{0}^{t} \operatorname{tr}\left[H\left(t^{\prime}\right)\right] d t^{\prime}},
$$

where $H$ is the Hamiltonian of the system. The trace of $H$ only contributes a complex multiplier to the evolution operator. Obviously the trace affects the required resources, but these resources are rather trivial as they amount to adding a common phase or attenuation to all states. In the first stage we are only interested in resources associated with actual state transformation. Therefore, to avoid the trivial changes in $U$ generated by the trace of $H$ we define the traceless Hamiltonian:

$$
\mathcal{H}=H-\frac{\operatorname{tr}(H)}{N} I .
$$

The evolution operator $\mathcal{U}$ generated by this Hamiltonian satisfies:

$$
\operatorname{det}[\mathcal{U}(t)]=1 \text {, }
$$


and it is related to $U$ through:

$$
\mathcal{U}=U / \operatorname{det}[U]^{1 / N}
$$

$\mathcal{U}$ is the determinant normalized version of $U$. The geometric meaning of condition (4) is given in appendix I. Despite (4), note that $\mathcal{U}$ is in general not a unitary operator:

$$
\mathcal{U}^{\dagger} \mathcal{U} \neq \mathrm{I}
$$

where $\nmid$ stands for the complex transposition operation. Assume there is some nonHermitian Hamiltonian $H(t)$ that after some time $T$ generates a desired evolution $U(T)$. Our goal will be to use $U(T)$ to set limits on the smallness of $H$. However, a simple scaling argument shows that for any final $U$, the generating Hamiltonian elements can be made arbitrarily small by making the evolution slower. To remove this ambiguity we will examine the time integral over the Hamiltonian "size". The scalar quantity we shall use to quantify the Hamiltonian size at each instant is the matrix norm of the Hamiltonian matrix. In summary, our goal is to set a lower bound on the action-like quantity:

$$
\int_{0}^{T}\|H(t)\| d t
$$

We shall refer to this quantity as the "norm action". In this work $\|\cdot\|$ refers to any matrix norm that satisfies the sub-multiplicative property:

$$
\|A B\| \leq\|A\|\|B\|,
$$

In particular, we shall often use the spectral norm [5] of the Hamiltonian. A previous study [6] showed that the spectral norm of $\mathcal{H}$ constitutes an upper bound on the evolution speed of a quantum state driven by $\mathrm{NH}$ or Hermitian Hamiltonian. This physical meaning of the spectral norm makes it our default choice of norm. Nonetheless, some of the results presented here equally apply to any sub-multiplicative norm just as well. Therefore the spectral norm will be used only where it can provide some additional insights unique to this norm. The (sub-multiplicative) spectral norm of the Hamiltonian is the largest singular value of $H$ [7] and it is equal to:

$$
\|H\|_{s}=\sqrt{\max \left[\operatorname{eig}\left\{H^{\dagger} H\right\}\right]},
$$

where eig \{\} is just the list of eigenvalues of the enclosed operator. Before we proceed, it is worth mentioning two properties of the spectral norm which provide more explicit information on the Hamiltonian matrix elements. Let $H_{i, j}$ be the elements of an operator $H \in \mathbb{C}^{N \times N}$. The spectral norm satisfies:

$$
\begin{aligned}
\|H(t)\|_{s} & \leq \sqrt{\sum_{i, j=1}^{N}\left|H_{i, j}\right|^{2}} \\
\max \left|H_{i, j}\right| \leq\|H(t)\|_{s} & \leq N \max \left|H_{i, j}\right|
\end{aligned}
$$

That is, a lower bound on (17) would also constitute a lower bound on $\int_{0}^{T} \sqrt{\sum_{i, j=1}^{N}\left|H_{i, j}(t)\right|^{2}} d t$ and on $\int_{0}^{T} \max \left|H_{i, j}(t)\right| d t$.

$\ddagger$ Loosely speaking the angular speed in "Bloch Sphere" 


\section{A lower bound on the resources needed for non-unitary evolution}

In this section we derive a lower bound on the "norm action" (7). The derivation is carried for the more general $H$ and $U$ and only later replace them by $\mathcal{H}$ and $\mathcal{U}$. The evolution operator $U(t)$ satisfies:

$$
i \frac{d}{d t} U=H U
$$

Taking the norm and using sub-multiplicativity (8):

$$
\left\|\frac{d}{d t} U\right\|=\|H U\| \leq\|H\|\|U\|
$$

or:

$$
\|H\| \geq\|U\|^{-1}\left\|\frac{d}{d t} U\right\|
$$

Since any norm satisfies the "inverse triangle inequality": $\|A-B\| \geq|\|A\|-\|B\||$, we get:

$$
\begin{aligned}
\left\|\frac{d}{d t} U\right\| & =\lim _{d t \rightarrow 0} \frac{\|U(t+d t)-U(t)\|}{d t} \\
& \geq\left|\frac{\|U(t+d t)\|-\|U(t)\|}{d t}\right|=\left|\frac{d}{d t}\|U(t)\|\right|
\end{aligned}
$$

Therefore:

$$
\|H\| \geq\|U\|^{-1}\left|\frac{d}{d t}\|U(t)\|\right| \geq\|U\|^{-1} \frac{d}{d t}\|U(t)\|
$$

Integrating we get our basic resources bound:

$$
\int_{0}^{T}\|H(t)\| d t \geq \ln \|U(T)\|
$$

An alternative derivation is given in appendix II.

Although this equation is correct, it is sensitive to $\operatorname{tr}(H)$ and $\operatorname{det}[U]$ which are related to the somewhat irrelevant information discussed earlier. This can be easily

resolved by noticing that $\mathcal{H}$ and $\mathcal{U}$ also satisfy $i \frac{d}{d t} \mathcal{U}=\mathcal{H} \mathcal{U}$, so (17) can be used to obtain:

$$
\int_{0}^{T}\|\mathcal{H}(t)\| d t \geq \ln \|\mathcal{U}(T)\|
$$

One may be concerned that perhaps $\|\mathcal{U}(T)\| \leq 1$ and therefore the RHS of (18) is negative which makes Eq. (18) trivial and useless. Nonetheless, for the spectral norm, one can show that:

$$
\|\mathcal{U}(T)\|_{s}=1 \Leftrightarrow \mathcal{U} \text { is unitary }
$$

Thus, for non-unitary operators the RHS of (18) is larger than zero and our lower bound is non-trivial. A simple derivation (19) is given in appendix III. Using the following property of the spectral norm:

$$
\|A\|_{s} \geq \max |\operatorname{eig}\{A\}|
$$


For any operator $A \in \mathbb{C}^{N \times N}$, it is possible to obtain a weaker lower bound in terms of the eigenvalues of evolution operator:

$$
\int_{0}^{T}\|\mathcal{H}(t)\|_{s} d t \geq \ln [\max |\operatorname{eig}\{\mathcal{U}\}|]
$$

In our third example we will examine a non-diagonalizable case ( $\mathrm{NH}$ degeneracy) where all the eigenvalues are equal to one so that the RHS of (21) is equal to zero. Nonetheless, the operator is still non-unitary and the stronger resources lower bound (18) is greater than zero.

The fact that the RHS of (18) is equal to zero for unitary evolution operators (due to (18) and (19)) gives some indication that our spectral norm bound quantifies the resources needed for non-unitary evolution but not the resources for unitary evolution. This point will be clarified in Sec. 5 However, before doing that we wish to extend our result to better fit the common case where the trace of the Hamiltonian is non-zero (e.g. a dissipative system).

\section{Improved inequality and the marginally passive case}

While losses are very common in physical systems, gain requires some special devices, special materials, power sources, etc. Therefore it is often easier to implement nonunitary evolution in passive systems (zero gain) at the expense of smaller signals at the output of the system (e.g. see the discussion in [9]). As will be explained next, the basic result (17), does not provide any useful information for passive systems as the inequality is trivially satisfied. The goal of this section is to generalize the previous results and remedy this problem.

Mathematically, a passive system cannot increase the norm of an input state. At most it can preserve it. In principle there is no point in attenuating the signal more than needed to reach passiveness. We shall use the term "marginally passive evolution" to describe a passive system that has at least one state whose norm is unchanged by $U$, while the norm of other states is either left unchanged or reduced. By definition the maximal norm of an output vector is given by the spectral norm of the transformation $U$. The final amplitude of a state $\left|\psi_{f}\right\rangle$ generated by $U$ from a normalized initial state $\left|\psi_{i}\right\rangle$ is simply: $\sqrt{\left\langle\psi_{f} \mid \psi_{f}\right\rangle}=\sqrt{\left\langle\psi_{i}\left|U^{\dagger} U\right| \psi_{i}\right\rangle}$. In fact, an alternative definition of the spectral norm is given by:

$$
\max _{\left|\psi_{i}\right\rangle} \sqrt{\left\langle\psi_{i}\left|U^{\dagger} U\right| \psi_{i}\right\rangle}=\|U\|_{s}
$$

Therefore, given a general non-unitary $U$, the marginally passive (MP) evolution operator is given by:

$$
U_{M P}=\frac{U}{\|U\|_{s}} .
$$

Since $\left\|U_{M P}\right\|_{s}=1$, and more generally for passive evolution $\left\|U_{P}\right\|_{s} \leq 1$, it becomes obvious that (17) is trivially satisfied for any passive evolution (LHS is positive while the RHS is negative or zero). In what follows we improve (17) to account for the passive system as well.

Inspection of (17) reveals that there is an unnatural asymmetry in the RHS. The "magnitude" of the non-unitary operation is determined by the maximal amplification (maximal singular value) while the maximal attenuation is completely ignored. 
Although in practice amplification is much more difficult to set up in comparison to attenuation, from a mathematical point of view, they should be treated on an equal footing. This asymmetry manifests itself clearly in the MP case. The amplification is zero and all the non-unitary information is in the degree of attenuation of state. To extract the attenuation information we will consider the inverse operation $U^{-1}$. The logic behind doing so, is that amplitude attenuation when going forward in time is equivalent to amplification when going backwards in time. We start by taking the time derivative of $U^{-1} U=I$ form which we get:

$$
\partial_{t} U=-U\left(\partial_{t} U^{-1}\right) U
$$

upon using it in the Schrödinger Eq. (12) we get:

$$
-i \partial_{t} U^{-1}=U^{-1} H
$$

Taking the norm on both sides and using sub-multiplicativity as we did before, we get:

$$
\left\|\partial_{t} U^{-1}\right\| \leq\left\|U^{-1}\right\|\|H\|
$$

Repeating the same procedure used to derive (17) we obtain:

$$
\int_{0}^{T}\|H\| d t \geq \ln \left\|U^{-1}\right\|
$$

Combining it with (17) we get the main result of this paper:

$$
\int_{0}^{T}\|H\| d t \geq \ln \left[\max \left\{\|U\|,\left\|U^{-1}\right\|\right\}\right]
$$

Since $\|U\|_{s}\left\|U^{-1}\right\|_{s} \geq 1$, for passive systems $\left\|U_{P}^{-1}\right\|_{s} \geq 1 /\left\|U_{P}\right\|_{s} \geq 1$. If so, the attenuation in the system provides a nontrivial result via the $U^{-1}$ term. Equation (28) resolves the aforementioned asymmetry between gain and loss. Later on it will be shown more explicitly for the spectral norm.

There is another way to combine the (17) and (27) which is less efficient than (28) (i.e. a smaller lower bound), but it offers some insights into the interplay of gain and loss and a more analytical structure. By adding (17) and (27) and dividing by two we get:

$$
\int_{0}^{T}\|H\| d t \geq \ln \sqrt{\|U\|\left\|U^{-1}\right\|}
$$

The norm of $U$ in (17) is replaced by the geometric mean of the norms of $U$ and $U^{-1}$. Moreover this expression is invariant under changes of the determinant of $U$ (or $\operatorname{tr} H$ ) since $\operatorname{det} U=1 / \operatorname{det} U^{-1}$.

The results (28) and (29) apply to any sub-multiplicative norm. Now we wish to focus on the spectral norm. It is easy to show that the singular values, $s$, of $U$, are just the inverse of the singular values of $U^{-1}$ (provided that $U$ is invertible). Therefore:

$$
\int_{0}^{T}\|H\|_{s} d t \geq \max \left\{s_{\max }, s_{\text {min }}^{-1}\right\} \geq \ln \sqrt{s_{\text {max }} s_{\text {min }}^{-1}}
$$

where $s_{\max }$ and $s_{\min }$ are the largest and smallest singular values of $U$. Here the symmetry between gain and loss appears more explicitly.

In the marginally passive case $\left\|U_{M P}\right\|_{s}=1$ so:

$$
\int_{0}^{T}\left\|H_{M P}\right\|_{s} d t \geq \ln \left\|U_{M P}^{-1}\right\|_{s}
$$


Finally, to conclude our discussion on the passive case we would like to point out a general feature of passive systems. It concerns the tradeoff between "nonUnitary performance" and "detectability". As shown in appendix I, $|\operatorname{det} U|^{1 / N}$ can be interpreted as the geometric average of the state amplifications generated by $U$. Starting from $U_{M P}$ and using (5) we get:

$$
\begin{aligned}
\|\mathcal{U}\|_{s} & =\left\|U_{M P} / \operatorname{det}\left[U_{M P}\right]^{1 / N}\right\|_{s} \\
& =\left\|U_{M P}\right\|_{s} /\left|\operatorname{det} U_{M P}\right|^{1 / N} \\
& =1 /\left|\operatorname{det} U_{M P}\right|^{1 / N}
\end{aligned}
$$

Combining this with the expression for the geometric mean amplitude, $\langle\langle\alpha\rangle\rangle$, from appendix I we get:

$$
\left\langle\left\langle\alpha_{M P}\right\rangle\right\rangle=\operatorname{det}\left[U_{M P}\right]^{1 / N}=\frac{1}{\|\mathcal{U}\|_{S}} \leq 1
$$

This clearly shows the performance vs. detectability tradeoff in the marginally passive case. The "stronger" the non-unitary operation, is the bigger $\|\mathcal{U}\|$ is. Yet, as $\|\mathcal{U}\|$ goes up, the geometric mean amplification goes down. In other words, if the system has no gain then stronger non-unitary operation will lead to a weaker signals.

\section{5. "Purely non-unitary" evolution and unitarily invariant norms}

In 8 a somewhat similar formula to (18) was written for unitary evolution. It follows from [8] that in the unitary case our $\ln \|\mathcal{U}(T)\|$ is replaced by $\|\ln \mathcal{U}(T)\|$. Unfortunately the appealing derivation in 8 , is suited only for Hermitian Hamiltonians and the analysis cannot be easily extended to NH Hamiltonians and non-unitary evolution. Our derivation overcomes this difficulty (also up until now we did not require that the norm be unitarily invariant), at the expense of the capability to capture the resources associated with unitary evolution. This can actually be an advantage if indeed our result always captures only the resources needed for the "purely non-unitary" part of the evolution. In this section we clarify in what sense and under what conditions our result (18) quantifies only the purely non-unitary part of the evolution operator.

For simplicity let us assume that $\mathcal{U}(T)$ is diagonalizable and that $\left\{\mu_{i}\right\}$ are the eigenvalues of the evolution operator. If all eigenvalues satisfy $\left|\mu_{i}\right|=1$, as in the unitary case, the information on the evolution is encapsulated in $\operatorname{Arg}\left[\mu_{i}\right]$. However if the eigenvalues are real and positive (and not all of them equal to one), it is clear the evolution is "purely non-unitary". The eigenstates are just attenuated or amplified without accumulating any phase as in the unitary case. With this in mind, we can make use of the singular value decomposition (SVD). According to the SVD scheme, any operator $U(T) \in \mathbb{C}^{N \times N}$ (even if it is not diagonalizable) can be decomposed into [7:

$$
\mathcal{U}(T)=Q W P^{\dagger}
$$

where $Q$ and $P$ are some unitary matrices $\Theta$ and $W$ is a diagonal non-negative matrix whose entries are the singular values mentioned earlier. In the unitary case $W$ is the identity matrix. In the non-unitary case $W$ corresponds to a purely non-unitary evolution operator. Equation (21) shows that any evolution can be written as a

$\S$ The exact forms of $P$ and $Q$ are not needed for our purpose. 
product of unitary evolution followed by a purely non-unitary evolution and then by another unitary evolution. Until now we did not require that the norm be unitarily invariant (UI). However, unitary invariance facilitates a very clear interpretation of the results. A norm is UI if it satisfies:

$$
\|A\|_{U I}=\|C A D\|_{U I}
$$

for any $A \in \mathbb{C}^{N \times N}$ and any unitary $C$ and $D$. The spectral norm, for example, is unitarily invariant norm. Using this property and Eq. (33) we get :

$$
\ln \|\mathcal{U}(T)\|_{U I}=\ln \|W\|_{U I}
$$

That is, the unitary part of evolution is removed and the lower bound we found addresses only the purely non-unitary part of the evolution.

\section{Examples}

In this section we demonstrate a few points about the resources inequality through explicit examples.

\subsection{Example 1 - A simple decay}

Consider a two-level time-independent Hamiltonian with one decaying level and one stable level:

$$
\begin{aligned}
& H=\left(\begin{array}{cc}
0 & 0 \\
0 & -i \Gamma
\end{array}\right) \\
& U=\left(\begin{array}{cc}
e^{0} & 0 \\
0 & e^{-\Gamma t}
\end{array}\right)
\end{aligned}
$$

The traceless Hamiltonian and the corresponding evolution operators are

$$
\begin{aligned}
& \mathcal{U}=\left(\begin{array}{cc}
e^{+\frac{1}{2} \Gamma t} & 0 \\
0 & e^{-\frac{1}{2} \Gamma t}
\end{array}\right) \\
& \mathcal{H}=\left(\begin{array}{cc}
+\frac{1}{2} i \Gamma & 0 \\
0 & -\frac{1}{2} i \Gamma
\end{array}\right)
\end{aligned}
$$

The norm action is:

$$
\int_{0}^{T}\|\mathcal{H}(t)\|_{s} d t=\frac{1}{2} \Gamma T
$$

The RHS of the resources inequality yield:

$$
\ln \|\mathcal{U}\|_{s}=\frac{1}{2} \Gamma T
$$

This elementary example demonstrates two important points. The first, is that the resources inequality (18) can be an exact equality in some cases. The resources inequality, then, is not an exaggerated overestimate. The second point is the importance of the trace removal and the $\mathcal{U}$ normalization. For $\Gamma>0$ it is easy to see that $\|U\|_{s}=1$ so $\ln \|U\|_{s}=0$ which make the resources inequality useless 
$\left(\int_{0}^{T}\left\|H_{0}(t)\right\|_{s} d t \geq 0\right)$. Alternatively, $\Gamma>0$ is exactly the marginally passive case so instead of normalizing $U$ we can use (28) and get:

$$
\begin{aligned}
& \int_{0}^{T}\left\|H_{0}(t)\right\|_{s} d t=\Gamma T \\
& \ln \left\|U_{0}^{-1}\right\|_{s}=\Gamma T
\end{aligned}
$$

Which is again an equality rather than an inequality.

\subsection{Example 2 - A cooling evolution}

Consider a quantum two-level system that has two solutions:

$$
\begin{aligned}
& \left|\psi_{1}\right\rangle=\left(\begin{array}{l}
1 \\
0
\end{array}\right), \\
& \left|\psi_{2}\right\rangle=\left(\begin{array}{l}
\sin \frac{\theta(t)}{4} \\
\cos \frac{\theta(t)}{4}
\end{array}\right),
\end{aligned}
$$

where $\theta(0)=0$. For simplicity we consider $\theta^{\prime}(t) \geq 0$ so that $\theta(t)$ is a monotonously increasing function. While the two solutions are initially orthogonal to each other, as $\theta \rightarrow \pi$ the two states become parallel to each other. This transformation changes a mixed state density matrix into a coherent one. Alternatively, this scheme can be used for non-orthogonal state discrimination. If one starts at $\theta(0) \neq 0$ and decreases the angle $\theta$, two initially non-orthogonal states become orthogonal when $\theta=0$. Once the states are orthogonal they can be resolved with $100 \%$ certainty. This kind of non-Hermitian state discrimination was first proposed in [3]. However, in [3] the needed resources were not considered. Moreover, in our example the Hamiltonian is time-dependent. The two solutions (44), (45) can be used to construct the evolution operator $U$ from which $\mathcal{U}$ and $\mathcal{H}$ can be explicitly calculated:

$$
\begin{aligned}
& \mathcal{H}=\frac{1}{2} i \theta^{\prime}(t)\left(\begin{array}{cc}
+\frac{1}{2} \operatorname{Tan}\left[\frac{\theta(t)}{2}\right] & 1 \\
0 & -\frac{1}{2} \operatorname{Tan}\left[\frac{\theta(t)}{2}\right]
\end{array}\right) \\
& \mathcal{U}=\frac{1}{\sqrt{\operatorname{Cos}\left[\frac{\theta(t)}{2}\right]}}\left(\begin{array}{cc}
1 & \operatorname{Sin}\left[\frac{\theta(t)}{2}\right] \\
0 & \operatorname{Cos}\left[\frac{\theta(t)}{2}\right]
\end{array}\right) \\
& \|\mathcal{U}\|_{s}=\sqrt{\frac{1+\sin \frac{\theta}{2}}{\cos \frac{\theta}{2}}}
\end{aligned}
$$

Now let us evaluate the norm action. The Hamiltonian norm is:

$$
\|\mathcal{H}\|_{s}=\frac{1}{4}\left|\theta^{\prime}\right| \frac{1}{\cos \left[\frac{\theta}{2}\right]}
$$

assuming monotonicity $\left(\theta^{\prime} \geq 0\right)$ :

$$
\int_{0}^{T}\|\mathcal{H}(t)\|_{s} d t=\frac{\theta}{4}+\frac{1}{2} \ln \frac{\cos \frac{\theta}{4}+\sin \frac{\theta}{4}}{\cos \frac{\theta}{4}-\sin \frac{\theta}{4}}
$$


After using few trigonometric identities we find that:

$$
\ln \|\mathcal{U}\|_{s}=\frac{1}{2} \ln \frac{1+\sin \frac{\theta}{2}}{\cos \frac{\theta}{2}}=\frac{1}{2} \ln \frac{\cos \frac{\theta}{4}+\sin \frac{\theta}{4}}{\cos \frac{\theta}{4}-\sin \frac{\theta}{4}}
$$

Equations (50) and (51) show that the resources inequality (18) holds $(\theta \geq 0)$. In this example the resources inequality was demonstrated for time-dependent Hamiltonians.

The resources divergence at $\theta \rightarrow \pi$ is a typical feature of non-unitary cooling and it can be understood without a detailed analysis. Since at the end of the evolution the two states become almost parallel to each other, the column vectors of $U$ become linearly dependent. As a result $\operatorname{det}[U] \rightarrow 0$. In order to to keep $\operatorname{det}[\mathcal{U}]=1$ (see ) the element of $\mathcal{U}$ must be very large. The LHS of Eq. (11) shows that if the elements of an operator are very large, the spectral norm of the operator is also very large.

The divergence at $\theta \rightarrow \pi$ seems to indicate that perfect cooling is not possible. Nonetheless, the divergence is only logarithmic, so in practice the norm action does not obtain large values in this case. For example, if $\theta=\pi-10^{-12}$, the minimal required resources is only about 15.3 in units of $\hbar$.

Nonetheless, the divergence is only logarithmic so in practice the norm action does not obtain large values in this case. For example, if $\theta=\pi-10^{-12}$ the minimal required resources is only about 15.3 in units of $\hbar$.

The comparison of $\mathrm{NH}$ cooling or state discrimination to other methods (relaxation via the Lindblad equation, POVM) is outside the scope of this work. Our goal is only to investigate fundamental limitation on the construction of non-unitary operations generated by the Schrödinger equation with NH Hamiltonians.

\subsection{Example 3 - Non-Hermitian degeneracy}

In this example we wish to examine the resources in the most extreme non-Hermitian scenario - the exceptional point Hamiltonian. Exceptional point Hamiltonians are Hamiltonians that cannot be diagonalized. At most, similarity transformations can bring these Hamiltonians to the standard canonical Jordan Block form. See Ch. 9 of [2] and references therein for more information on exceptional points. Consider the following exceptional point Hamiltonian and its corresponding evolution operator:

$$
\begin{aligned}
\mathcal{H} & =\left(\begin{array}{cc}
0 & E_{0} \\
0 & 0
\end{array}\right) \\
\mathcal{U} & =\left(\begin{array}{cc}
1 & -i E_{0} T \\
0 & 1
\end{array}\right)
\end{aligned}
$$

The norm action is:

$$
\int_{0}^{T}\|H(t)\|_{s} d t=\left|E_{0}\right| T
$$

Since $U^{-1}$ is just $U$ with $E_{0} \rightarrow-E_{0}$, the bound based on $U^{-1}$ gives the same result as the bound based on $U$. The RHS of the resources inequality is:

$$
\ln \|U\|_{s}=\ln \sqrt{1+\frac{1}{2}\left|E_{0}\right| T\left(\left|E_{0}\right| T+\sqrt{4+\left|E_{0}\right|^{2} T^{2}}\right)}
$$

If the resources inequality holds we should expect:

$$
e^{2\left|E_{0}\right| T} \geq 1+\frac{1}{2}\left|E_{0}\right| T\left(\left|E_{0}\right| T+\sqrt{4+\left|E_{0}\right|^{2} T^{2}}\right),
$$


Next we wish to show analytically that this inequality indeed holds.

$$
\begin{aligned}
e^{2\left|E_{0}\right| T} & =\sum \frac{\left(2\left|E_{0}\right| T\right)^{n}}{n !} \geq 1+\left|E_{0}\right| T+\left(\left|E_{0}\right| T\right)^{2} \\
& =1+\frac{1}{2}\left|E_{0}\right| T\left(\left|E_{0}\right| T+2+\left|E_{0}\right| T\right) \\
& \geq 1+\frac{1}{2}\left|E_{0}\right| T\left(\left|E_{0}\right| T+\sqrt{4+\left|E_{0}\right|^{2} T^{2}}\right)
\end{aligned}
$$

This example shows that in some cases the eigenvalues of $\mathcal{U}$ are completely inadequate to describe the dynamics. In this example eig $\{\mathcal{U}\}=\{1,1\}$ so the RHS of (21) is equal to zero and therefore the eigenvalues of $\mathcal{U}$ carry no new or useful information on the resources integral. This is similar to the finding in [6], the evolution speed near $\mathrm{NH}$ degeneracies in the Hamiltonian is determined by the spectral norm of the Hamiltonian and not by the eigenvalues of the Hamiltonian. Moreover this example shows that time-independent Hamiltonian does not necessarily have a norm action which is equal to the bound as was the case in our first example.

\section{Concluding remarks}

It was shown that non-unitary evolution requires some minimal resources. The "norm action" of the Hamiltonian has to be larger than the norm of the evolution operator or its inverse. For a passive system it was also shown that larger non-unitary changes inevitably lead to weaker signals.

The spectral norm of the Hamiltonian was studied before in the context of evolution speed in non-unitary evolution [6. Moreover it was used to find the most efficient Hamiltonians for a given trajectory in the projective Hilbert space. Will these efficient Hamiltonian also give the minimal norm action (7)? Not necessarily. The set of constraints in this work is completely different. Here we consider the final state of all possible initial states while [6] concerns the resources optimization of a single specific state with a given evolution. Furthermore, in this work there is no constraint on the evolution between the beginning and the end of the evolution.

Our bound captures only the "pure non-unitary" part of the evolution. In 8 a norm action bound for unitary evolution and Hermitian Hamiltonians was introduced. It would be interesting to find a bound that exclusively captures the unitary part of the evolution (for non-unitaty evolution) as well as a bound that captures everything together and study the relations between the different bounds.

\section{Acknowledgments}

The author wishes to cordially thank Uwe Günther for the suggestion to investigate the marginally passive case. The generous hospitality of ITAMP where this work has been completed is greatly acknowledged.

\section{Appendix I - The geometric mean amplification}

We define the mean amplification in the following way: let $\{|m\rangle\}_{m=1}^{N}$ be an orthogonal set under the standard inner product. The amplification of each element generated 
by an evolution operator $U$ is given by:

$$
\alpha_{m}=\sqrt{\frac{\left\langle m\left|U^{\dagger} U\right| m\right\rangle}{\langle m \mid m\rangle}}
$$

The geometric mean is:

$$
\alpha_{\text {avg }}=\sqrt[N]{\alpha_{1} \ldots \alpha_{N}}=\left(\Pi \sqrt{\operatorname{eig}\left\{U^{\dagger} U\right\}}\right)^{1 / N}=|\operatorname{det} U|^{1 / N}
$$

To illustrate the reasoning behind the geometric mean consider a two-level system with two orthogonal eigenstates. One is amplified by a factor of two and the other by a factor of one-half. The algebraic mean is equal to $5 / 4$ while the geometric mean is equal to one. The geometric mean, then, matches our expectation that the two and one-half should balance each other. If so, the requirement $|\operatorname{det} \mathcal{U}|=1$ (or equivalently

that $\left.\int_{0}^{t_{f}} \operatorname{tr}(H) d t^{\prime}=0\right)$ used in section (3), actually means that the geometric mean amplification is equal to one.

\section{Appendix II}

Given some time dependent Hamiltonian $H(t)$ the evolution operator at some time $T$ can be written as:

$$
U(T)=\lim _{d t \rightarrow 0} e^{-i H(T) d t} . . e^{-i H\left(t_{j}\right) d t} \ldots e^{-i H\left(t_{1}\right) d t} e^{-i H\left(t_{0}\right) d t},
$$

where $t_{j}=j d t$. Using the sub-multiplicativity and the basic norm properties we get:

$$
\|U(T)\| \leq \lim _{d t \rightarrow 0}\left\|e^{-i H(T) d t}\right\| \ldots\left\|e^{-i H\left(t_{j}\right) d t}\right\| \ldots\left\|e^{-i H\left(t_{0}\right) d t}\right\| .
$$

Moreover, each term satisfies:

$$
\begin{aligned}
\left\|e^{-i H\left(t_{j}\right) d t}\right\|= & \left\|\sum_{k=0}^{\infty} \frac{\left(-i H\left(t_{j}\right) d t\right)^{k}}{k !}\right\| \leq \sum_{k=0}^{\infty} \frac{\left\|\left(-i H\left(t_{j}\right) d t\right)^{k}\right\|}{k !} \\
& \leq \sum_{k=0}^{\infty} \frac{\left\|H\left(t_{j}\right)\right\|^{k} d t^{k}}{k !}=e^{\left\|H\left(t_{j}\right)\right\| d t}
\end{aligned}
$$

Therefore:

$$
\|U(T)\| \leq \lim _{d t \rightarrow 0} e^{\|H(T)\| d t} \ldots e^{\left\|H\left(t_{j}\right)\right\| d t} \ldots e^{\left\|H\left(t_{0}\right)\right\| d t}
$$

Now the RHS is a multiplication of numbers so:

$$
\|U(T)\| \leq e^{\lim _{d t \rightarrow 0} \sum_{j}\left\|H\left(t_{j}\right)\right\| d t}=e^{\lim _{d t \rightarrow 0} \sum_{j}\left\|H\left(t_{j}\right)\right\| d t}
$$

From which we once again obtain the resources inequality:

$$
\int_{0}^{T}\|H\| d t \geq \ln \|U(T)\|
$$


Resources needed for non-unitary quantum operations

\section{Appendix III}

In this appendix, for completeness, we give a simple derivation (19):

$$
\|\mathcal{U}(T)\|_{s}=1 \Leftrightarrow \mathcal{U} \text { is unitary }
$$

One direction is trivial: if $\mathcal{U}$ is unitary so that $\mathcal{U}^{\dagger} \mathcal{U}=I$, then all the singular values are equal to one. In particular the largest singular value (the spectral norm) is equal to one.

For the other direction we use the fact that $\operatorname{det} \mathcal{U}=1$. From this we get that:

$$
\operatorname{det} \mathcal{U}^{\dagger}=\left(\operatorname{det} \mathcal{U}^{T}\right)^{*}=1
$$

From which follows:

$$
\prod s_{i}^{2}=\operatorname{det} \mathcal{U}^{\dagger} \mathcal{U}=\operatorname{det} \mathcal{U}^{\dagger} \operatorname{det} \mathcal{U}=1
$$

where $s_{i} \geq 0$ are the singular values of $\mathcal{U}$ (the square root of $\operatorname{eig}\left\{\mathcal{U}^{\dagger} \mathcal{U}\right\}$ ). If $\|\mathcal{U}(T)\|_{s}=\max \left\{s_{i}\right\}=1$ then to satisfy (68) it must be that all the singular value are equal to one. Thus, the SVD decomposition of the $\mathcal{U}$ is:

$$
\mathcal{U}=Q W P^{\dagger}=Q P^{\dagger}
$$

where $\mathrm{Q}$ and $\mathrm{P}$ are unitary matrices and therefore:

$$
\mathcal{U}^{\dagger} \mathcal{U}=P Q^{\dagger} Q P^{\dagger}=I
$$

which completes the proof.

[1] Zheng C, Hao L, Long G L 2011 arXiv:1105.6157 [quant-ph]

[2] Moiseyev N, Non-Hermitian Quantum Mechanics, (Cambridge University Press, Cambridge, 2011).

[3] Bender C M, Brody D C, Caldeira J, and Meister B K, 2010 arXiv:1011.1871 1 [hep-th]

[4] Chicone C, Ordinary Differential Equations with Applications 1st edn. (NewYork: Springer, 1999)

[5] Horn R A and Johnson C R, Matrix analysis, (Cambridge: Cambridge University Press, 1985).

[6] Uzdin R, Günther U, Rahav S and Moiseyev N 2012 J. Phys. A: Math. Theor. 45415304

[7] Horn R A and Johnson C R, Topic in Matrix Analysis, (Cambridge: Cambridge University Press, 1991).

[8] Lidar D A, Zanardi P, and Khodjasteh K 2008 Phys. Rev A 78, 012308

[9] Günther U and Samsonov B F, 2008 Phys. Rev. Lett 101230404 